\title{
ON THE TWO-REALIZABILITY OF CHAIN COMPLEXES
}

\author{
SUSHIL JAJODIA
}

\begin{abstract}
We give a sufficient condition which insures the realizability of a two-dimensional chain complex satisfying Wall's condition by a two-dimensional CW-complex.
\end{abstract}

Let $\pi$ be a group generated by $x_{1}, \ldots, x_{n}$. Let $C_{*}$ be a two-dimensional chain complex

$$
\begin{array}{cccccc}
C_{*}: & C_{2} & \stackrel{\partial_{2}}{\rightarrow} & C_{1} & \stackrel{\partial_{1}}{\rightarrow} & C_{0} \\
& & \| & & \| \\
Z \pi^{m} & & Z_{\pi^{n}} & & Z_{\pi}
\end{array}
$$

satisfying these conditions: (i) $H_{1}\left(C_{*}\right)=0$, (ii) $H_{0}\left(C_{*}\right) \simeq Z$, and (iii) the boundary operator $\partial_{1}=\left(x_{1}-1, \ldots, x_{n}-1\right)$. In [7], Wall conjectured that under the above conditions $C_{*}$ could be realized as the homology chains of a two-dimensional $\mathrm{CW}$-complex. However, in [3], Dunwoody gave an example of a chain complex $C_{*}$ which satisfied Wall's conditions but was not realizable. The purpose of this note is to give a sufficient condition which insures the realizability of $C_{*}$ by a two-dimensional CW-complex.

Suppose there is a presentation $\mathscr{P}=\left(x_{1}, \ldots, x_{n}: R_{1}, \ldots, R_{m}\right)$ for the group $\pi$ (see the Remark 2 below). We let $F=F\left(x_{1}, \ldots, x_{n}\right)$, the free group generated by $x_{1}, \ldots, x_{n}$ and $R=N_{F}\left\{R_{1}, \ldots, R_{m}\right\}$, the normal closure in $F$ of $R_{1}, \ldots, R_{m}$. Let $\bar{F}=F\left(r_{1}, \ldots, r_{m}\right)$, the free group on symbols $r_{1}, \ldots, r_{m}$, and let $\psi: F * \bar{F} \rightarrow F$ be the homomorphism taking $x_{i} \mapsto x_{i}$ and $r_{j} \mapsto R_{j}$.

Now if $\varphi: F \rightarrow F / R=\pi$ is the natural projection and $\kappa: F \rightarrow C_{1}$ is the crossed homomorphism, then by Corollary 4.4, p. 655 of [8], we can find words $W_{1}, \ldots, W_{m}$ such that the matrix $\left\|\kappa\left(W_{i}\right)\right\|^{\varphi}$ is the boundary operator $\partial_{2}$. The problem is that $N_{F}\left\{W_{1}, \ldots, W_{m}\right\}$ may not generate the entire normal subgroup $R$. Because each $W_{i} \in R$, we can write

$$
W_{i}=\prod_{k=1}^{m_{i}}\left(x_{i k} R_{i k} x_{i k}^{-1}\right)^{\varepsilon_{i k}}
$$

where $R_{i k}=R_{j}$, for some $j, \quad 1<j \leqslant m, \quad x_{i k} \in F, \varepsilon_{i k}= \pm 1$. Let $w_{i}=$ $\Pi_{k=1}^{m_{i}}\left(x_{i k} r_{i k} x_{i k}^{-1}\right)^{e_{i k}}$ where if $R_{i k}=R_{j}$ in $W_{i}$, then $r_{i k}=r_{j}$ in $w_{i}$. Let $J$ denote the

Received by the editors May 30, 1979 and, in revised form, August 17, 1979.

AMS (MOS) subject classifications (1970). Primary 55A05, 20 F05. 
$m \times m$ matrix

$$
J=\left\|\begin{array}{ccc}
\frac{\partial w_{1}}{\partial r_{1}} & \cdots & \frac{\partial w_{1}}{\partial r_{m}} \\
\vdots & & \\
\frac{\partial w_{m}}{\partial r_{1}} & \cdots & \frac{\partial w_{m}}{\partial r_{m}}
\end{array}\right\| .
$$

Now we prove

LEMMA. The following are equivalent.

(1) $N_{F}, \bar{F}\left\{w_{1}, \ldots, w_{m}\right\}=N_{F}, \bar{F}\left\{r_{1}, \ldots, r_{m}\right\}$.

(2) $\left\{x_{1}, \ldots, x_{n}, w_{1}, \ldots, w_{m}\right\}$ forms a generating set for $F * \bar{F}$.

(3) $J$ has a right inverse.

Proof. (1) $\Rightarrow(2)$ is obvious.

(2) $\Rightarrow$ (3). Suppose $\left\{x_{1}, \ldots, x_{n}, w_{1}, \ldots, w_{m}\right\}$ forms a generating set for $F * \bar{F}$. Then by the Inverse Function Theorem [1], the Jacobian which has the form

$$
\left\|\begin{array}{ll}
I_{n} & 0 \\
A & J
\end{array}\right\|
$$

has a right inverse $B$. By a result of Kaplansky [6], $B$ is a two-sided inverse so that $J$ has a right inverse.

(3) $\Rightarrow(1)$. Suppose $J$ has a right inverse $H$. Then the Jacobian which has the form

$$
\left\|\begin{array}{cc}
I_{n} & 0_{m} \\
A_{m \times n} & J_{m}
\end{array}\right\|
$$

has a right inverse

$$
\left\|\begin{array}{cc}
I_{n} & 0_{m} \\
-H_{m} A_{m \times n} & H_{m}
\end{array}\right\| .
$$

Thus, by the Inverse Function Theorem $\left\{x_{1}, \ldots, x_{n}, w_{1}, \ldots, w_{m}\right\}$ is a generating set for $F * \bar{F}$. We claim $\left.N_{F, \bar{F}}\left\{w_{1}, \ldots, w_{m}\right\} \stackrel{\text { def }}{=} \bar{N}=N \stackrel{\text { def }}{=} N_{F *}, r_{1}, \ldots, r_{m}\right\}$. Clearly $\bar{N} \subseteq N$. Therefore we have the short exact sequence $1 \rightarrow N / \bar{N} \rightarrow F * \bar{F} / \bar{N}$ $\rightarrow F * \bar{F} / N \rightarrow 1$. Since $F * \bar{F} / \bar{N} \cong F$ and $F * \bar{F} / N \cong F$, we must have $N=\bar{N}$. This completes the proof of the lemma.

REMARK 1. Let $J^{\alpha}$ denote the image of $J$ under the abelianizing homomorphism $\alpha$ acting on $Z(F * \bar{F})$. Then $N_{F, \bar{F}}\left\{w_{1}, \ldots, w_{m}\right\}=N_{F \cdot \bar{F}}\left\{r_{1}, \ldots, r_{m}\right\}$ only if the determinant $\operatorname{det} J^{\alpha}$ is a unit in $Z(F * \bar{F})^{\alpha}$. For $N_{F \cdot \bar{F}}\left\{w_{1}, \ldots, w_{m}\right\}=$ $N_{F, \bar{F}}\left\{r_{1}, \ldots, r_{m}\right\}$ implies that $\left\{x_{1}, \ldots, x_{n}, w_{1}, \ldots, w_{m}\right\}$ is a basis for $F * \bar{F}$. By Corollary 2 of [1], det $\bar{J}$ is a unit in $Z(F * \bar{F})^{\alpha}$ where

$$
\bar{J}=\left\|\begin{array}{ll}
I_{n} & 0 \\
A & J
\end{array}\right\|
$$

therefore $\operatorname{det} \bar{J}=\operatorname{det} J$ is a unit in $Z(F * \bar{F})^{\alpha}$. 
THEOREM. Notation as above. The chain complex $C_{*}$ is realizable by a two-dimensional $C W$-complex if the Jacobian $J$ has a right inverse.

Proof. By the above lemma, we have $N_{F, \bar{F}}\left\{w_{1}, \ldots, w_{m}\right\}=N_{F \cdot \bar{F}}\left\{r_{1}, \ldots, r_{m}\right\}$. Applying $\psi$ we get $N_{F}\left\{W_{1}, \ldots, W_{m}\right\}=R$. Therefore if $R$ denotes the cellular model of the presentation $R=\left(x_{1}, \ldots, x_{n}: W_{1}, \ldots, W_{m}\right)$ for $\pi$, then the universal cover $\tilde{R}$ of $R$ realizes the given chain complex $C_{*}$.

REMARK 2. It is possible that there does not exist a presentation $\mathscr{P}$ with $n$ generators and $m$ relators. This would happen if the relation module $\bar{R}=\operatorname{ker} \partial_{1}$ is generated by fewer than $m$ elements. (See Dyer [4].)

REMARK 3. Because both

$$
\left\{x_{1}, \ldots, x_{n}, w_{1}, \ldots, w_{m}\right\} \text { and }\left\{x_{1}, \ldots, x_{n}, r_{1}, \ldots, r_{m}\right\}
$$

form generating sets for the free group $F * \bar{F}$, we can convert one set to the other using Nielsen transformations (see [5]). This implies that $P$ and $R$ which are the cellular models of the presentations $\mathscr{P}$ and $\mathscr{R}$, respectively, have the same simple homotopy type.

ExAMPLE. Let $\pi$ be the group $Z_{5} \times Z_{5} \times Z_{5}$ generated by $a, b$, and $c$, and let $C_{*}$ be the chain complex

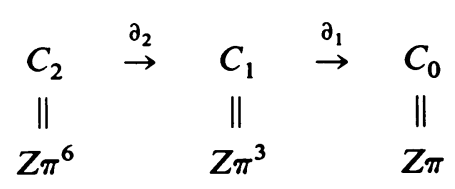

where $\partial_{1}=(a-1, b-1, c-1)$ and $\partial_{2}$ is the matrix

$$
\left\|\begin{array}{ccc}
\frac{a^{5}-1}{a-1} & 0 & 0 \\
0 & \frac{b^{5}-1}{b-1} & 0 \\
0 & 0 & \frac{c^{5}-1}{c-1} \\
1-b & a-1 & 0 \\
1-c & 0 & a-1 \\
0 & 1-c & b-1
\end{array}\right\| .
$$

Let $\mathscr{P}$ be the presentation $\left(a, b, c: a^{5}, b^{5}, c^{5},\left[a^{4}, b\right],[a, c],[b, c]\right)$. We see that we can take $W_{1}=a^{5}, W_{2}=b^{5}, W_{3}=c^{5}, W_{4}=a b a^{-5} b^{-1}\left[a^{4}, b\right]^{-1} a^{5} a^{-1}, W_{5}=[a, c]$, and $W_{6}=[b, c]$ and the corresponding Jacobian $J$ has a right inverse. Therefore the chain complex $C_{*}$ is realizable. Indeed,

$$
\Re=\left(a, b, c: a^{5}, b^{5}, c^{5},[a, b],[a, c],[b, c]\right),
$$

and the cellular models $P$ and $R$ have the same simple homotopy type. 


\section{REFERENCES}

1. J. S. Birman, An inverse function theorem for free groups, Proc. Amer. Math. Soc. 41 (1973), 634-638.

2. W. Cockcroft and R. M. F. Moss, On the two-dimensional realizability of chain complexes, J. London Math. Soc. 11 (1975), 257-262.

3. M. J. Dunwoody, Relation modules, Bull. London Math. Soc. 4 (1972), 151-155.

4. M. N. Dyer, Spaces dominated by finite two-dimensional CW-complexes (to appear).

5. W. Magnus, A. Karrass and D. Solitar, Combinatorial group theory, Pure and Appl. Math., vol. 13, Interscience, New York, 1966.

6. M. S. Montgomery, Left and right inverses in group algebras, Bull. Amer. Math. Soc. 75 (1969), 539-540.

7. C. T. C. Wall, Finiteness conditions for CW-complexes. II, Proc. Roy. Soc. Ser. A 295 (1966), 129-139.

8. R. C. Lyndon, Cohomology theory of groups with a single defining relation, Ann. of Math. (2) 52 (1950), 650-665.

Department of Mathematics, University of OKLahoma, Norman, OKLahoma 73019

Current address: Department of Mathematics and Computer Science, University of Wisconsin, Stevens Point, Wisconsin 54481 